\title{
Measuring Compassion in Healthcare: A Comprehensive and Critical Review
}

\author{
Shane Sinclair ${ }^{1}$ Lara B. Russell ${ }^{2,3} \cdot$ Thomas F. Hack $^{4,5} \cdot$ Jane Kondejewski $^{1}$. \\ Richard Sawatzky,
}

Published online: 19 November 2016

(c) Springer International Publishing Switzerland 2016

\begin{abstract}
Background There is international concern about the lack of compassion in healthcare systems. A valid and reliable tool for measuring compassion in healthcare systems and educational institutions is required. This comprehensive and critical narrative synthesis identified and compared existing measures of compassionate care in clinical settings.

Methods PubMed, MEDLINE, CINAHL and PsycINFO databases and grey literature were searched to identify studies that report information on instruments that measure compassion or compassionate care in clinicians, nurses, healthcare students and patients. Textual qualitative descriptions of included studies were prepared. Instruments were evaluated using the Evaluating Measures of PatientReported Outcomes (EMPRO) tool.

Results Nine studies containing information on the Compassion Competence Scale, a self-report instrument that measures
\end{abstract}

Electronic supplementary material The online version of this article (doi:10.1007/s40271-016-0209-5) contains supplementary material, which is available to authorized users.

Shane Sinclair

sinclair@ucalgary.ca

1 Faculty of Nursing, University of Calgary, 2500 University Drive NW, Calgary, AB T2N 1N4, Canada

2 School of Nursing, University of Victoria, Victoria, BC, Canada

3 Centre for Health Evaluation and Outcome Sciences, Providence Health Care, Vancouver, BC, Canada

4 Manitoba Palliative Care Research Unit, CancerCare Manitoba, Winnipeg, MB, Canada

5 Rady Faculty of Health Sciences, College of Nursing, University of Manitoba, Winnipeg, MB, Canada

6 School of Nursing, Trinity Western University, Langley, BC, Canada compassion competence among Korean nurses; the Compassion Scale, the Compassionate Care Assessment Tool $\odot$, and the Schwartz Center Compassionate Care Scale ${ }^{\mathrm{TM}}$, patient-reported instruments that measure the importance of healthcare provider compassion; the Compassion Practices Scale, an instrument that measures organisational support for compassionate care; and instruments that measure compassion in educational institutions (instructional quality and a Geriatric Attitudes Scale), were included. Each instrument is associated with significant limitations. Most only measure certain aspects of compassion and lack evidence of adaptability to diverse practice settings. The EMPRO of self-report instruments revealed a lack of psychometric information on measurement reliability, validity, responsiveness and interpretability, respondent, administrative and scoring burden, and use in subpopulations.

Conclusion The findings of this narrative synthesis identified an unmet need for a psychometrically validated instrument that comprehensively measures the construct of compassion in healthcare settings.

\section{Key Points for Decision Makers}

Compassionate care is an essential feature of quality healthcare that is lacking in many healthcare systems and educational institutions.

Measurement validation is required for clinical measures of compassion to evaluate, improve and further clinical practice, quality of healthcare and patient reported outcomes.

Available instruments are associated with significant limitations, and there remains an unmet need for a clinically relevant and psychometrically robust measure of compassion in healthcare. 


\section{Introduction}

Compassion has been variously defined as a feeling, attitude or trait that arises in witnessing another's distress and that motivates a subsequent desire to help [1-3]. Compassion is an inherently relational care construct, engendering and developing relationships to achieve relevant outcomes aimed at addressing needs, specifically those related to suffering [4-6]. In clinical settings, compassion actuates healthcare providers to provide care that focuses on a patient's humanity and uniqueness [4]. Healthcare encounters that include compassion can offer substantial benefits for patients, such as increasing trust in the patientphysician relationship, improving patient satisfaction, symptoms and quality of life, and aiding recovery [1, 5, 7-17]. Compassion can also have a positive effect on clinician outcomes through increased job satisfaction and retention [5].

In several studies, patients and their families have identified compassion as a necessity during healthcare encounters [7]. A recent systematic review of compassion in healthcare [5] identified compassion as a patient right [18], central to codes of ethics across healthcare professions [19-21], a practice competency that should be evaluated in selecting medical and nursing student admissions, $[22,23]$, a standard of care that healthcare providers must be trained in and systems must measure and report $[11,17,24]$ and a dimension of quality care that should be assessed on an ongoing basis [17, 25]. Despite these efforts, there is persistent evidence that a lack of compassion is a key contributor to failures within healthcare systems $[11,17,21,25,26]$ and that receiving compassionate healthcare remains an important but unmet patient need $[8,10,11,14,17]$.

A significant barrier to improving and furthering clinical practice, quality care and patient satisfaction is the lack of a clinical measure of compassion with strong evidence of measurement validity [27]. The need for a scientifically sound measure of compassion was acknowledged in 2008 by the Royal College of Nursing in the UK, but a validated compassion measure as a necessary and recognised component of assessing healthcare quality and the patient-reported care experience is still unavailable [16]. While some patient satisfaction and experience measures such as the Consumer Assessment of Healthcare Providers and Systems Programme in USA and the National Health Service Adult In-Patient Survey in England evaluate aspects of compassion and have been modified and purported as compassion measures [14, 27], they do not directly address the construct of compassion, including its multiple dimensions.
Research investigating how patients understand and experience compassion in clinical care is beginning to emerge [6]; however, a recent systematic review identified that less than one-third of studies on the topic involve patients [5]. The limited studies in patient cohorts have identified compassion in clinical care as a consortium of skills, including listening, confronting, involvement, helping, presence, understanding and communicating [28, 29], in addition to a feeling, attitude or trait. An empirical model developed in advanced cancer patients revealed virtues, relational space, virtuous response, seeking to understand, relational communication and attending to needs aimed at relieving suffering as key dimensions of compassion [6]. Based on patient accounts, this study generated the following evidence-based definition of compassion: a virtuous response that seeks to address the suffering and needs of a person through relational understanding and action. The same research team discovered that patients were able to distinguish between and prefer compassion in comparison to related concepts of sympathy and empathy [30]. As the nature of compassion in clinical care becomes more evident, it is to be expected that the construct of compassion will be further delineated and become easier to measure. Although clinical measures of compassion have been proposed, comprehensive and critical reviews of validity evidence pertaining to tools for measuring compassion in clinical care are scarce. The objectives of this current review of the literature were to (1) identify how compassionate care is being measured in clinical settings, (2) provide a comparative review of existing measurement instruments to reveal gaps in the evidence base and (3) provide recommendations that will inform future research, clinical practice and policy in the measurement of compassionate care in healthcare.

\section{Methods}

A narrative synthesis approach was used, which involves a systematic review and synthesis of findings from multiple studies and relies primarily on the use of words and text to summarise and discuss the findings [31]. The narrative synthesis framework consisted of four elements: (1) developing a research question: what is the current state and quality of compassion measures in healthcare?; (2) developing a preliminary synthesis of findings; (3) exploring the relationships between studies; and (4) assessing the robustness of the synthesis. In addition, a comparative review of the measurement instruments was conducted using a validated standardised tool for 'Evaluating the Measurement of Patient-Reported Outcomes' (EMPRO) [32]. 


\subsection{Search Strategy}

A review team (SS, RS, TH, LR, JK) comprising measurement and content experts was compiled to ensure the review process was appropriate, comprehensive and rigorous. The team developed a search strategy to identify specific studies that have measured compassion or compassionate care in a clinical setting. One of the authors (JK) conducted a search of the literature using the electronic databases PubMed, MEDLINE, CINAHL and PsycINFO, under the direction of the lead author (SS). The initial search was broad to ensure wide coverage of the topic and included the search terms compassion, compassionate care, measure, instrument, scale, model and tool. In a second search, the scope was narrowed to identify instruments that have been used to measure compassion or compassionate care in a clinical setting. The search term compassion was combined with a pre-existing search filter that was developed and validated for the purpose of finding studies on psychometric properties of measurement instruments in PubMed [33]. In addition, grey literature searches of relevant organisational websites (e.g. Schwartz Center for Compassionate Healthcare) and Google Scholar were completed, and a manual search of the reference lists from relevant articles was carried out. Forward citation searches of included studies using Web of Science were also conducted. Searches were restricted to studies in the English language published between 1985 and 2016.

\subsection{Inclusion and Exclusion Criteria}

Studies were included in the final synthesis if they reported on instruments for the measurement of compassion or compassionate care in samples of clinicians, nurses, healthcare students and patients. Exclusion criteria were: (1) qualitative or mixed-method studies; (2) non-clinical settings (e.g., community, healthy populations); (3) studies that focused on related concepts such as empathy, sympathy, pity, self-compassion compassion fatigue, fear of compassion and compassion satisfaction; (4) neurological and neuroplasticity research that reported psychophysiologic changes in response to non-verbal communication of compassion; and (5) letters, commentaries, editorials, conference abstracts and case studies.

\subsection{Study Selection}

Two review authors (SS, JK) examined titles and abstracts to select eligible studies. The full text of potentially relevant studies was retrieved and examined to determine which studies met the inclusion criteria. Disagreements about study selection were resolved by discussion with the review team until consensus was reached.

\subsection{Data Extraction and Management}

A review author (JK) extracted data from eligible studies. Information was collated in a tabular form, including first author's last name, year of publication, study objective, study design, setting, participants, context, methods of statistical analysis, results and notes. Studies were classified based on types of measurement instruments (clinician-reported instruments; patient-reported instruments; measures of organisational support for compassionate care; measuring compassion in educational institutions).

\subsection{Data Synthesis}

A narrative synthesis approach was used. Textual descriptions and qualitative case descriptions of included studies were prepared. The review team met regularly to discuss the process and results of the review. The review team identified emerging groups or clusters, modifying study implementation and the results accordingly.

\subsection{Comparative Review of the Measurement Instruments}

EMPRO is a validated tool for the assessment of patientreported outcomes that was used to evaluate the psychometric properties of the instruments included in this study. It consists of 38 items assessing the evidence regarding various psychometric properties of a measure (reliability, validity, responsiveness, interpretability, administrator and respondent burden, alternative modes of administration). Ratings are made on a 4-point scale ('strongly disagree' to 'strongly agree'), and are based on information reported in sources such as research literature, reports and user manuals. Subscale scores and an overall EMPRO score can be computed [32].

Two authors (JK, LR) independently reviewed the relevant publications for each instrument and completed a first round of EMPRO ratings. Differences between the ratings were reviewed and discussed until a full consensus was reached (between three and four 'rounds' per instrument).

\section{Results}

The searches identified 1300 articles. Titles and abstracts were screened, and 91 studies were considered potentially eligible for inclusion. After analysing the full text of the 91 studies, 82 studies were excluded. Nine studies describing seven instruments were found eligible for inclusion in this review (Fig. 1). 


\subsection{Characteristics of Included Studies}

The characteristics of the included studies are shown in Table 1. One study described a self-report instrument for identifying compassion competence among practicing nurses in Korea [34]. Four studies described patient-reported instruments to examine the importance of physician and nurse compassion in different patient populations [35-38]. One study that measured organisational support for compassionate care was also included [39]. Three studies measured compassion in educational institutions, either as indicators of educator instructional performance and quality, or as attributes in residents, fellows or medical/ nursing students [40-42]. Table 2 summarises the characteristics of the compassion and compassionate care assessment instruments as reported in the included articles.

\subsection{Clinician-Report Instruments}

\subsubsection{Compassion Competence Scale}

Lee et al. [34] developed and psychometrically evaluated the Compassion Competence Scale as a tool for measuring compassion competence among practicing nurses in Korea. This scale is self-administered by nurses and attempts to measure behaviours that cause patients to perceive their
Fig. 1 Flow diagram of search strategy

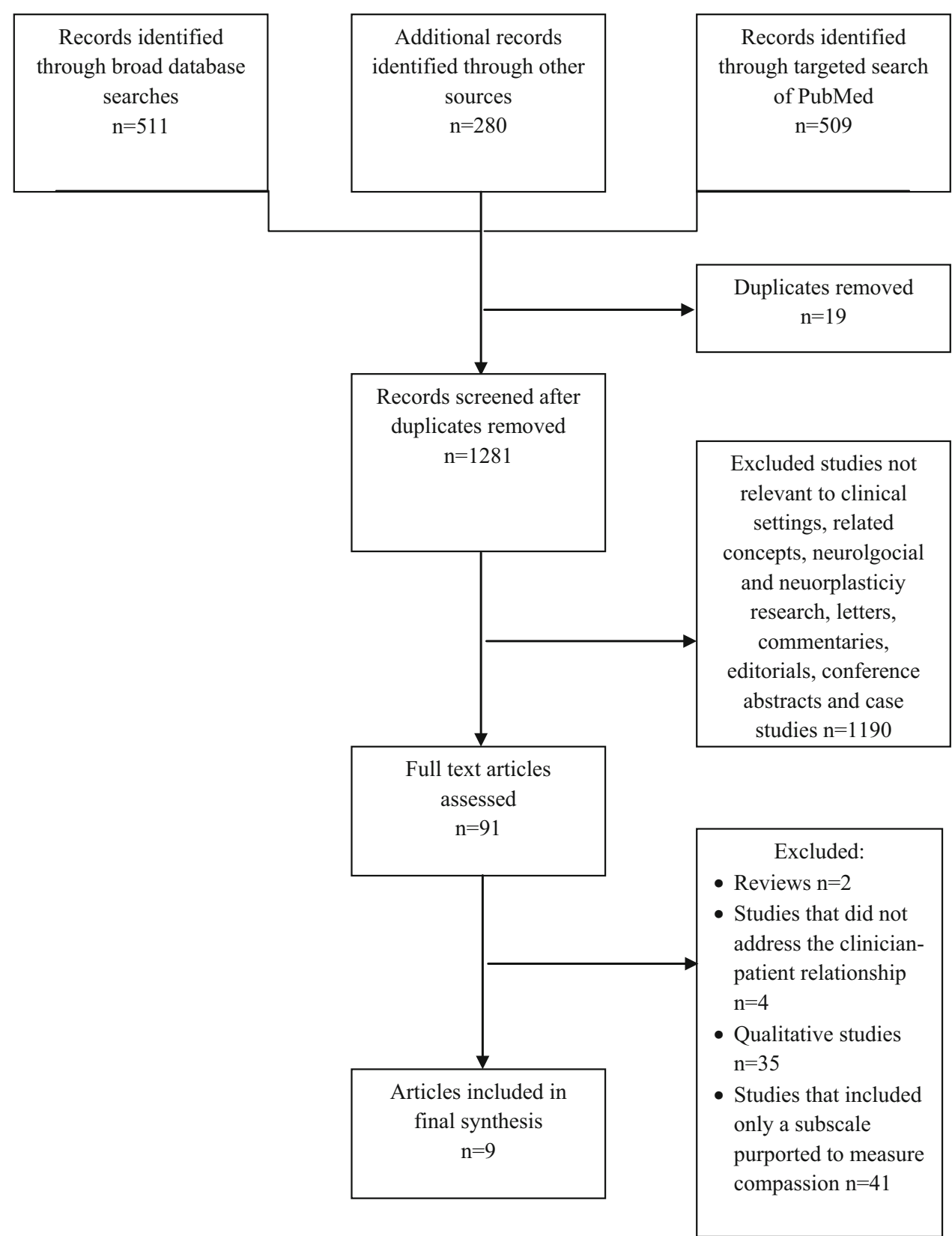




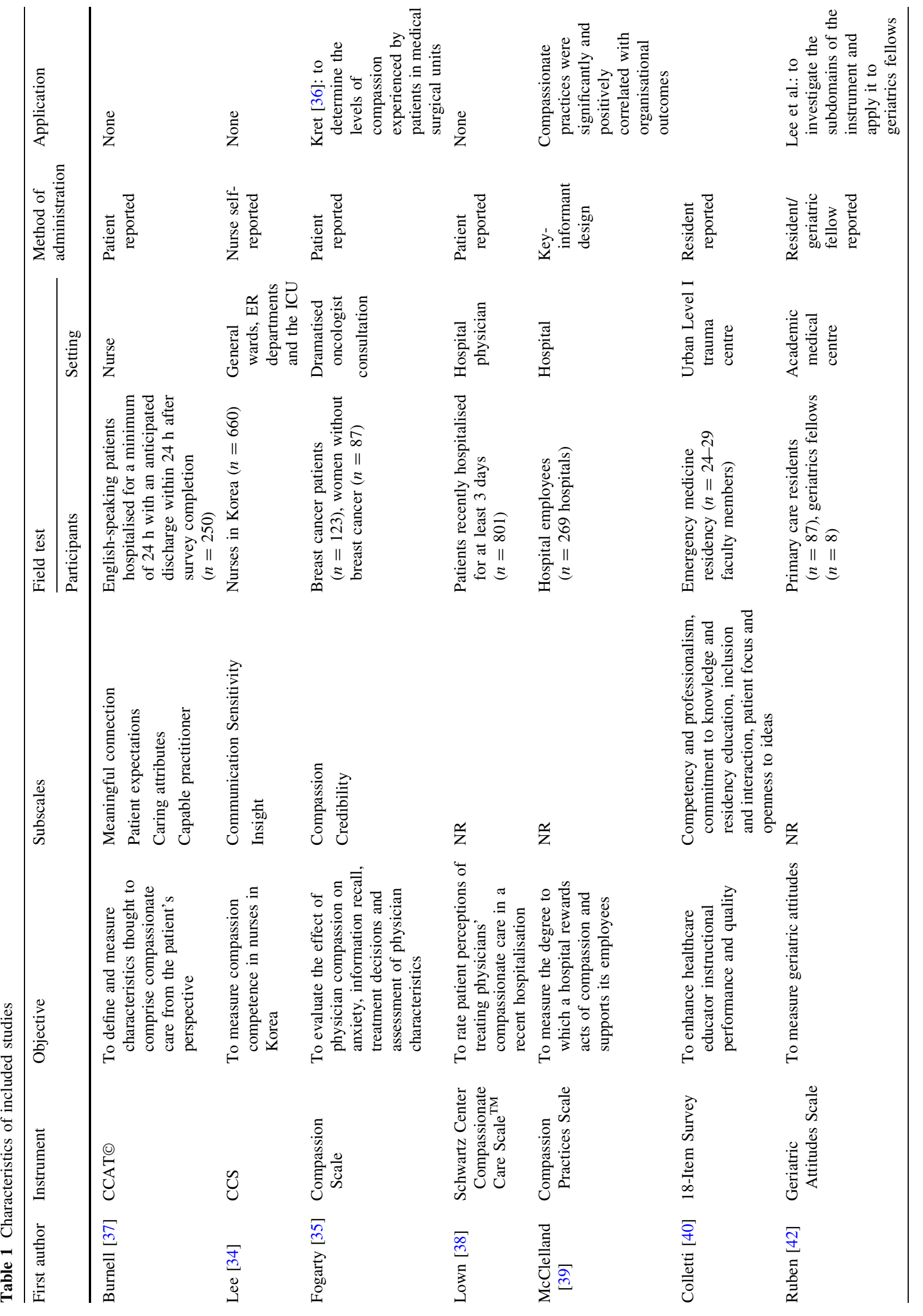




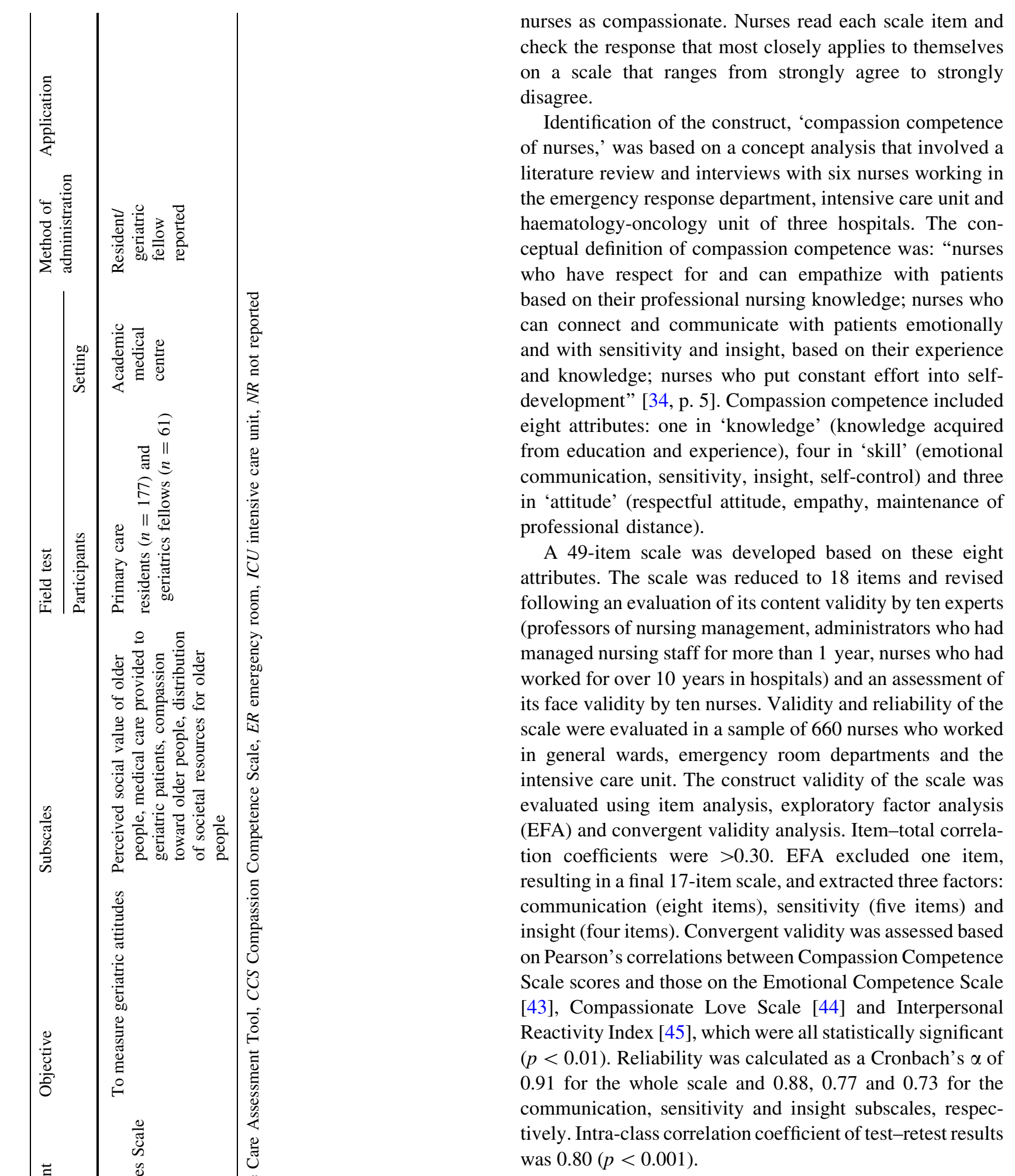

nurses as compassionate. Nurses read each scale item and check the response that most closely applies to themselves on a scale that ranges from strongly agree to strongly isagree.

Identification of the construct, 'compassion competence of nurses,' was based on a concept analysis that involved a literature review and interviews with six nurses working in the emergency response department, intensive care unit and haematology-oncology unit of three hospitals. The conceptual definition of compassion competence was: "nurses who have respect for and can empathize with patients based on their professional nursing knowledge; nurses who can connect and communicate with patients emotionally and with sensitivity and insight, based on their experience development" [34, p. 5]. Compassion competence included eight attributes: one in 'knowledge' (knowledge acquired rom education and experience), four in 'skill' (emotional in 'attitude' (respectful attitude, empathy, maintenance of rofessional distance) following an evaluation of its content validity by ten experts (professors of nursing management, administrators who had its face validity by ten nurses. Validity and reliability of the in general wards, emergency room departments and the . analysis (EFA) and convergent validity analysis. Item-total correla(1tem, insight (four items). Convergent validity was assessed based Pearson's correlations between Compassion Competence cale scores and those on the Emotional Competence Scale 43], Compassionate Love Scale [44] and Interpersonal Reactivity Index [45], which were all statistically significant $(\rho<0.01)$. Reliability was calculated as a Cronbach's $\alpha$ of 0.91 for the whole scale and $0.88,0.77$ and 0.73 for the was $0.80(p<0.001)$

\subsection{Patient-Reported Instruments}

\subsubsection{Compassion Scale}

Fogarty et al. [35] developed a videotape stimulus to evaluate the effect of patients' perceptions of physician 


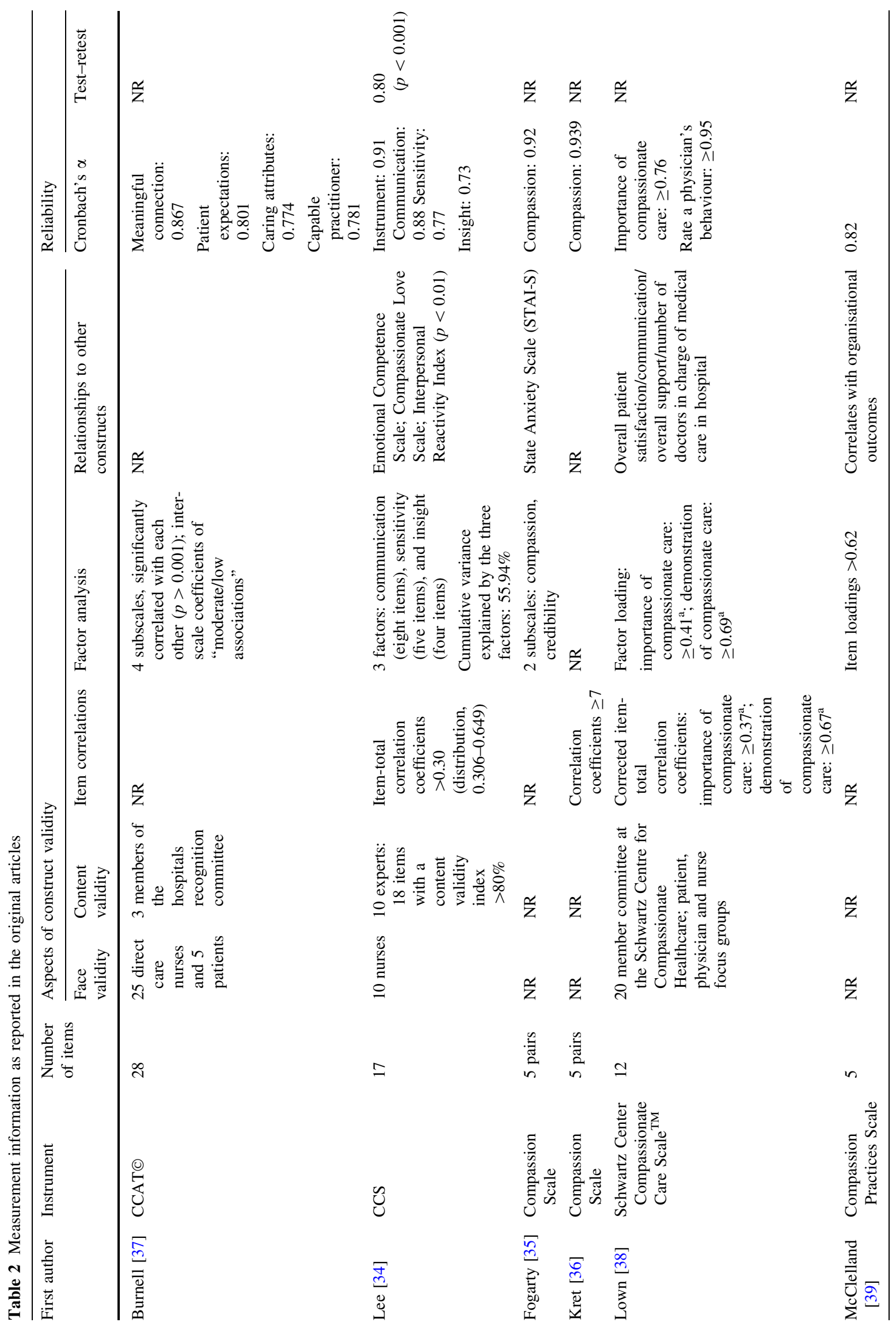




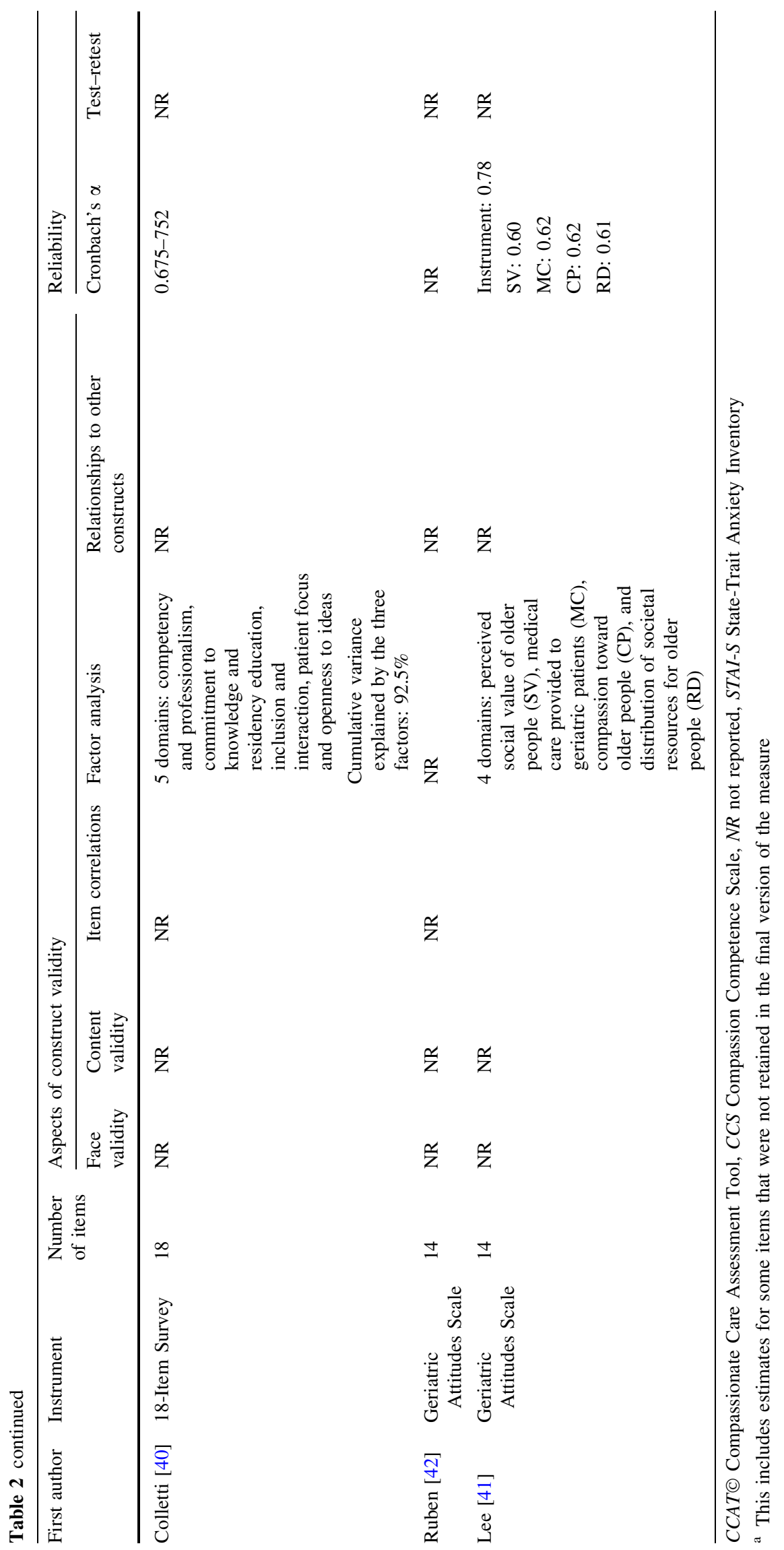


compassion on anxiety, information recall, treatment decisions and assessment of physician characteristics in female breast cancer survivors and healthy women without cancer. For their study, Fogarty et al. [35] applied a dictionary definition of compassion as "Sympathetic concern for the suffering of another, together with the inclination to give aid or support or show mercy" (as cited in [35] p. 372). Two videos of a dramatised oncologic consultation were created. In the standard video, the physician gave the patient information on treatments for metastatic breast cancer and prognosis. In the enhanced compassion video, the physician provided the same information, but the actors used words and touch to express support, sympathy and compassion for the patients. Patients rated the physicians' compassion on a visual analogue scale ranging from 0 to 100 for each of the following five pairs of characteristics: cold/warm, unpleasant/pleasant, distant/compassionate, insensitive/sensitive and uncaring/caring. The sum of the five items indicated the degree to which study participants perceived the physician as compassionate, with higher scores indicating a greater level of compassion.

Psychometric evaluation of the Compassion Scale involved interviews with women who had watched the compassion enhanced video to identify items that measured the degree to which the physician displayed compassion. Factor analysis resulted in two subscales, the 'compassion' subscale (five items) and the 'credibility' subscale (four items). The compassion subscale had good evidence of internal consistency reliability with a Cronbach's $\alpha$ of 0.92 [35]. Validity of the compassion subscale was further examined by comparing the scores of women who used it to evaluate physician compassion as demonstrated in the enhanced compassion video or the standard video. The enhanced compassion video received significantly higher scores overall and for each item of the compassion subscale.

Fogarty's Compassion Scale was applied by Kret et al. [36] to determine the levels of compassion experienced by patients in medical surgical units. One hundred nurses were rated on their compassion by 100 patients, with each patient rating one nurse. In this study, the Compassion Scale had a Cronbach's $\alpha$ of 0.939 , and inter-item Spearman correlation coefficients were all $\geq 0.7$, which suggested that all items had a strong relationship with each other.

\subsubsection{Compassionate Care Assessment Tool (CCAT)@}

Burnell et al. [37] developed the Compassionate Care Assessment Tool (CCAT) (C) to evaluate elements of compassionate nursing care in an acute hospital setting. This tool combines the constructs of compassion and caring to measure nursing behaviours and actions that are considered compassionate. Burnell et al. [37] defined compassion as "a sympathetic consciousness of others' distress with a desire to alleviate it" in accordance with Merriam-Webster, 2010 (as cited in [37], p. 181). This definition of compassion was broadened to include a spiritual context, based on the observation that major world religions consider compassion central to their beliefs and traditions. Caring was defined as "feeling and exhibiting concern and empathy for others," according to WordNet, 2010 (as cited in [37], p. 181).

Burnell et al. [36] attempted to identify, observe and measure the relationship between patients' spiritual needs, including compassion, and nurses' caring behaviour. The CCAT@ was derived from items within The Spiritual Needs Survey [46] and the Caring Behaviors Inventory (CBI) [47]. The Spiritual Needs Survey asks patients if they experienced a spiritual need in any of 28 areas, including compassion, during a present hospitalisation and to rate the importance of that need on a scale from slightly to extremely important [46]. The CBI asks patients to rate their nurse's caring process on a six-point Likert-type scale ranging from never to always [47].

The CCATC was developed following a pilot study conducted in 110 hospitalised patients in USA, in which patients were asked to complete both surveys. The 28 items of the CCAT $\odot$ were based on the ten highest scoring items from The Spiritual Needs Survey and the CBI, as well as the ten items that were most highly correlated to the compassion and kindness statement in the Spiritual Needs Survey and the ten items that were most highly correlated to the question asking patients to rate the concern nurses demonstrated to them in the CBI. Content validity of the CCATC was examined by three members of the hospital's recognition committee, which is responsible for presenting the DAISY ${ }^{\circledR}$ Award for Extraordinary Nurses, an honour that is awarded based on a number of criteria, including compassionate care. Face validity was assessed by 25 direct care nurses and five patients.

The CCATC was psychometrically evaluated in 250 patients in a hospital setting. Compassionate care was predefined for each patient as "understanding suffering and wanting to do something about it". Patients were asked to rate the personal importance of each CCATC item on a scale of 1 (not important) to 4 (extremely important). Factor analysis identified four subscales, including meaningful connection (eight items), patient expectations (five items), caring attributes (four items), and capable practitioner (three items). Cronbach's $\alpha$ was $0.87,0.81,0.77$ and 0.78 for the meaningful connection, patient expectations, caring attributes and capable practitioner subscales, respectively. 


\subsubsection{Schwartz Center Compassionate Care Scale ${ }^{T M}$}

Lown et al. [38] created the Schwartz Center Compassionate Care Scale ${ }^{\mathrm{TM}}$ to measure patient perceptions of the compassionate care provided by their treating physician during a recent hospitalisation. The scale, intended for research, educational assessment and quality performance improvement programmes, was developed using 16 items identified by a committee (20 cancer survivors, individuals experiencing chronic pain and/or debilitating illnesses, family members of patients, and individuals working in healthcare policy and advocacy) to evaluate the compassionate care provided by physicians and other caregivers for the purposes of adjudicating on a compassionate care award [14]. These items were vetted through focus groups (patient, nurse, physician) and incorporated into a larger national survey of patients' care experiences during a recent hospital admission. Survey participants were read a description of compassionate healthcare: "Now, I would like to turn to an approach to treating patients known as compassionate health care that focuses on improving the relationships between doctors, nurses and other professional caregivers and patients and their families. Its particular focus is to improve the communication and emotional support that patients receive from their doctors, nurses and other professional caregivers". Participants were asked to rate the personal importance of each item and the degree to which that behaviour had been demonstrated by their doctor during their most recent hospitalisation on a scale of 1, "Not at all important" (for questions related to importance) or "Not at all successfully demonstrated" (for questions related to demonstration) to 10, "Absolute priority; needs maximum attention" (importance) or "Very successfully" (demonstration) [14]. Evaluation of the responses referring to the importance of compassionate care revealed that the items were unidimensional with an acceptable Cronbach's $\alpha(>0.76)$. Similar findings were found when the items were used to rate a physician's behaviour, except the Cronbach's $\alpha$ was higher $(>0.95)$.

Based on data from the national survey, the scale was reduced to 12 items. Scale items correlated with patients' ratings of communication/emotional support provided by their doctor during the most recent hospitalisation, and were higher in participants who had only one doctor in charge of their medical care during the recent hospitalisation.

The Schwartz Center Compassionate Care Scale ${ }^{\mathrm{TM}}$ is currently available as a 12-item scale for use when measuring patients' perceptions of compassionate care provided by hospital physicians.

\subsection{Measures of Organisational Support for Compassionate Care}

\subsubsection{Compassion Practices Scale}

McClelland and Vogus [39] developed a Compassion Practices Scale to evaluate the extent to which a hospital uses award programmes to recognise acts of caring shown by employees' to patients, families and other employees, and the degree to which the hospital provides support for employees and departments to manage occupational stress. The scale comprises five items measured on a 7-point Likert-type scale. Top-level hospital executives from 269 non-federal acute care hospitals in USA were surveyed. Psychometric properties were evaluated. EFA showed all five items loaded onto a single factor with a Cronbach's $\alpha$ of 0.82. Compassionate practices were significantly and positively correlated with organisational outcomes, including patients' overall rating of the hospital and the likelihood of recommending the hospital to a friend or family member.

\subsection{Measuring Compassion in Educational Institutions}

A number of instruments that include one or more items for the measurement of compassion have been developed in educational contexts to enhance healthcare educator instructional performance and quality. For example, Colletti et al. [40] developed a structured and validated instrument to measure instructional quality in an emergency medicine residency in an urban Level I trauma centre in USA. An 18-item survey was administered to residents as a faculty evaluation instrument, containing one item that focused on compassionate patient care. Reuben et al. [42] developed and validated a Geriatric Attitudes Scale for primary care residents. This 14 -item scale aims to measure geriatric residents' attitudes towards patients on a 5 -point Likert scale where $1=$ least positive and $5=$ most positive. Lee et al. [41] extended the study to investigate the subdomains of the instrument and apply it to geriatrics fellows. They conducted factor analysis, which revealed four domains: (1) perceived social value of older people (SV); (2) medical care provided to geriatric patients (MC); (3) compassion toward older people (CP); and (4) distribution of societal resources for older people (RD). Cronbach's $\alpha$ was 0.78 for the overall instrument and $0.60,0.62$, 0.62 and 0.61 for SV, MC, CP and RD, respectively. The four subscales were correlated $(r=0.22-0.48)$, indicating each individual subscale measured a distinct dimension. The four subscales detected between- and within-group differences in residents and fellows over time. 


\subsection{Comparative Review of the Measurement Instruments}

Item and subscale scores for six of the seven measurement instruments are presented in Table 3. The faculty evaluation instrument [40] was not included as it contained only one item that focused on compassionate healthcare.

\subsubsection{Conceptual and Measurement Model}

Several of the instruments scored relatively high in this area, including the CCAT $\odot$ (52.38), Compassion Competence Scale (52.38), Compassion Scale (52.38) and the Schwartz Center Compassionate Care Scale ${ }^{\mathrm{TM}}$ (42.86). For all measures, the concept to be measured was well defined. However, evidence to support the proposed measurement model was mixed. For most of the measures, there was little information about scale variability or dimensionality.

3.6.1.1 Reliability Only two of the instruments, the Compassion Competence Scale and the Schwartz Center Compassionate Care Scale ${ }^{\mathrm{TM}}$, had sufficient data to compute subscale scores for reliability. Both instruments scored 41.67 for internal consistency. The Compassion Competence Scale was the only instrument with test-rest information and scored 16.67 for reproducibility. The other instruments did not receive reliability subscale scores owing to lack of information, but acceptable internal consistency coefficients were presented for two (Compassion Practices Scale and Compassion Scale). Reliability was evaluated using item response theory approaches only for the Schwartz Center Compassionate Care Scale ${ }^{\mathrm{TM}}$.

3.6.1.2 Validity The Schwartz Center Compassionate Care Scale $^{\mathrm{TM}}$ had the highest validity subscale score (58.33), followed by the Compassion Competence Scale (48.61) and CCAT( (13.89). The remaining instruments had too much missing data on the EMPRO validity items to support the calculation of a validity score.

3.6.1.3 Responsiveness, Interpretability, Burden and Alternative Modes Responsiveness and interpretability are subscale scores that are included in the EMPRO overall score, but none of the instruments had enough items to allow for calculation of these subscales. Similarly, none of the instruments had enough data to calculate a subscale score for respondent burden, and only the Compassion Competence Scale had sufficient data to allow computation of the subscale for 'administrative and scoring burden'. Only one instrument, the CCAT $\odot$, had evidence regarding alternative modes (self-administered and interviewer administered). Although some data about score differences between modes were provided, the implications of these differences were not discussed in the corresponding publication.

\section{Discussion}

The results of this systematic narrative review revealed that the availability of validated and reliable instruments that measure compassionate care in healthcare providers is poor. Our research identified one instrument that was developed to measure compassion as an emotion in healthcare providers $[35,36]$, three instruments that were created to measure healthcare providers' ability to provide compassionate care $[34,37,38]$, one study that measured compassion in association with the patient care experience [39] and three studies that evaluated compassion as indicators of educator instructional performance and quality, or as attributes in residents, fellows and medical/nursing students [40-42]. While we report on several measures of compassion, each is associated with significant limitations that warrant careful consideration.

The most significant limitation we discovered was related to conceptual issues, which in part is owing to an inherent challenge associated with measuring compassion-a complex relational construct that includes elements that are not easily measurable and vary according to individuals (both provider and recipient) and context [48]. These conceptual issues were rarely addressed, with predetermined definitions of compassion influencing item selection without conducting initial face and construct validation. A number of qualitative studies, for example, have identified common elements of compassion in healthcare such as virtues, listening, confronting, involvement, responding, helping, presence, understanding and communicating [5, 6, 28, 29, 49-51]. We recommend that a comprehensive measure of compassionate care should include qualitative elements identified by those individuals who are at the epicentre of the compassionate care interchange-patients and their healthcare providers. While qualitative elements of compassion present their own challenges they also attest to the dispositional aspects of compassion, and guard against the development of measures that strictly assess caring or compassionate behaviours. As such, while compassion is ultimately in the eye of the recipient (the patient), we recommend that a patientreported compassionate care measure should also be informed by qualitative research of healthcare provider understandings and experiences of providing compassionate care to provide a thorough understanding of these internal motivators and their expression in clinical practice. While innate and qualitative aspects of compassion, such as emotional resonance, virtues and presence, present their own challenges in measure development, a recent 


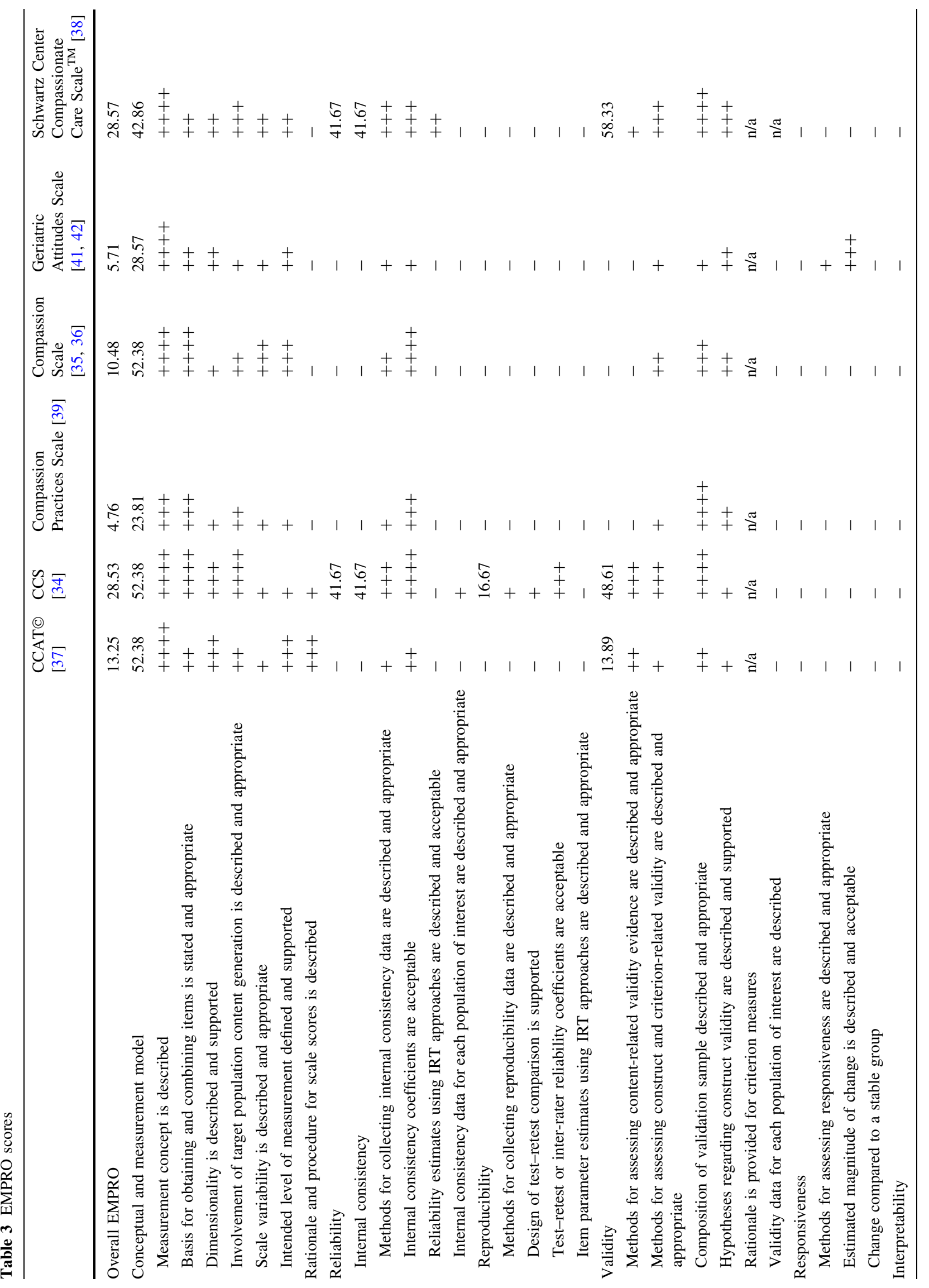




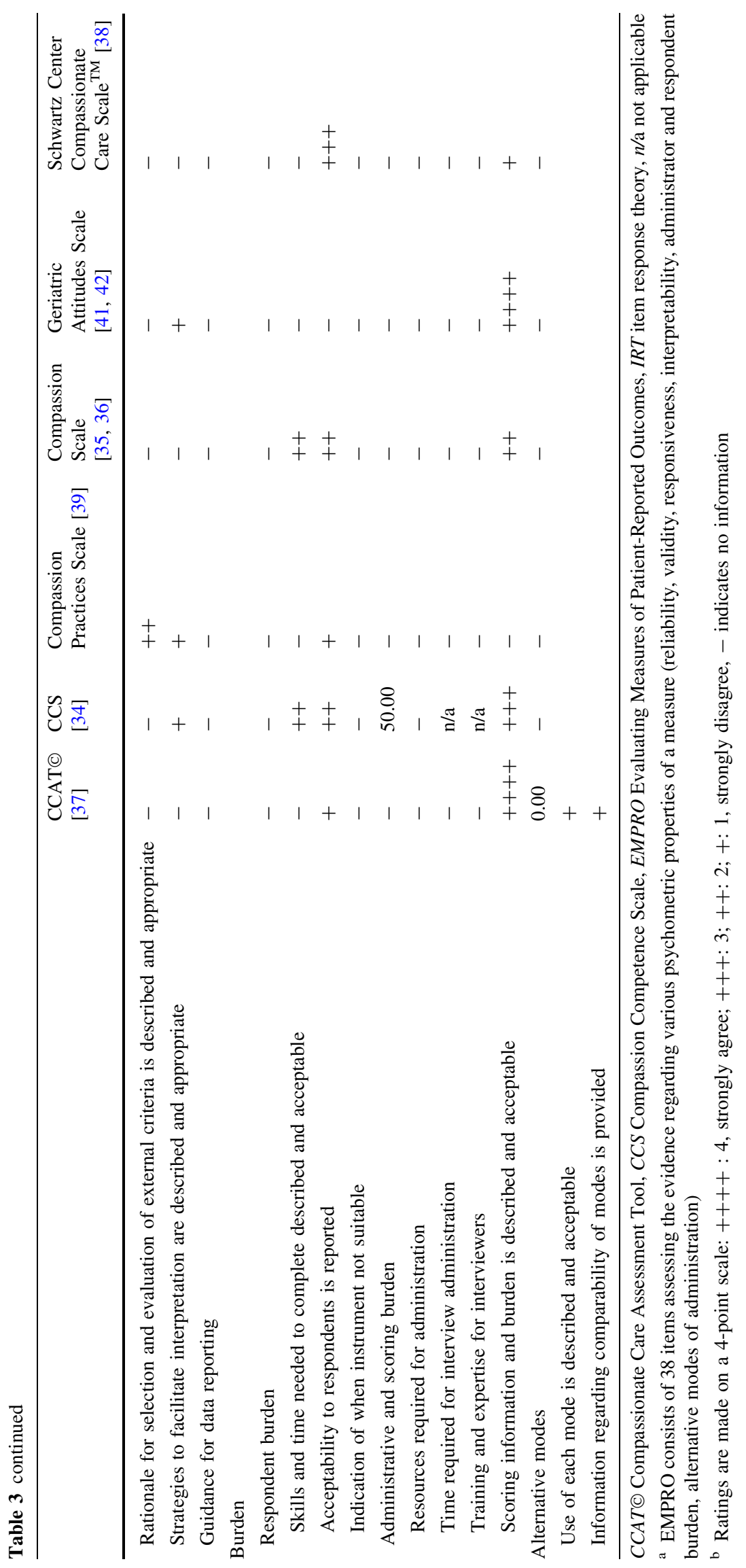


qualitative study of patients' understandings and experiences of compassion suggests that these qualitative domains are observable and essential in guarding against a prescriptive approach that strictly measures behaviours patients 'perceive' as compassionate [6].

Most of the measures included in this review only measured an aspect of compassion or used an a priori definition of compassion [37, 38, 40-42]. Some used dictionary definitions or conceptualisations based on literature searches, many of which included aspects of empathy or sympathy in their definitions of compassion [34-36]. These concepts have been differentiated from compassion, as compassion is distinct in terms of motivators (virtues vs. pity [sympathy] and emotions [empathy]) and outcomes (relief of suffering vs. self-preservation of the observer [sympathy] and understanding [empathy]) [5, 6]. In fact, the items in most instruments address empathy, with only the Compassion Competence Scale [34] including an item that directly measures the desire to help alleviate suffering, which is a key element that delineates compassion from the related construct of empathy [30]. A further limitation was that three studies included 'compassion' as a term in one of the items in their scale instead of providing an adjective describing a variable that facilitates assessment of compassion as a construct [35, 36, 38].

Among the instruments identified in this review, the Schwartz Center Compassionate Care Scale ${ }^{\mathrm{TM}}$ incorporated many of the elements of compassion reported in the literature [38]; however, it did not account for two distinguishing and central facets of the construct-its virtuebased motivators and its predication in action [6, 50, 51]. This scale was also limited in that it did not report details on the purpose or findings of the focus groups that the instrument(s) were vetted through; it used items modified from criteria for a compassionate care award; there was no initial construct or face validation performed on the construct itself; and it seemed to be a part of a larger survey of patient experience during a recent hospital admission (0-18 months prior to the survey) with no other details provided.

Finally, while the developers of the Schwartz Center Compassionate Care Scale $^{\mathrm{TM}}$ note that further development of the tool in "research, educational assessment and quality improvement programmes" is necessary [38], its applicability in clinical practice is not known. The Compassion Competence Scale [34] also addresses a number of core elements of compassion. The communication subscale assesses a nurse's interpersonal skills, while the sensitivity subscale measures a nurse's ability to recognise and react to changes in patients' emotions. The insight subscale evaluates a nurse's ability to use their professional knowledge to understand patients' needs and offer care. Unfortunately, the Compassion Competence Scale was developed on the premise that compassion is not directly observable or measurable. As such, the scale focuses on estimates of the effects of compassion demonstrated through particular behaviours, rather than directly on the dimensions or facets of compassion. In addition, the scale is self-administered by nurses and reports on their perceptions of their own skills related to compassion. As compassion involves relational and experiential aspects, the evaluation of compassion competence in healthcare providers should be based on a patient-informed model and evaluated in accordance with the guidelines for the development of self-reported measures [52-54].

Fogarty's [35] Compassion Scale is a patient-reported measure; however, it is limited as it only measures the affective aspects of compassion, and does not account for action. The scale does not directly measure interpersonal and relational factors, practical compassion or communication; rather, it evaluates perceptions of a clinical interaction from the perspective of an outside observer. The videotape stimulus is non-relational in nature and seems to focus on actions that are perceived as compassionate without accounting for more intuitive, affective and relational components. The CCATC [37] addresses several core variables that have been identified as acts of compassion, including virtues, communication, responding and helping; however, it does not assess understanding and it was developed by amalgamating select items from instruments that were originally designed to measure spiritual well-being and caring in general. Furthermore, the CCAT ( has yet to be used to measure compassionate care in a clinical sample. We recommend that a measure of compassionate care should include and assess core dimensions of compassion as reported by patients.

In addition to the above, it is important to note that none of these instruments have been used in diverse patient populations and healthcare settings. The Compassion Competence Scale was developed and tested in Korea in a single sample of nurses, and has not been used in any other studies. Lee et al. [34] state that it needs to be validated and tested in English across a variety of cultures and subjects to determine its potential to measure compassionate competence in other healthcare settings, providers and patient populations. Fogarty's Compassion Scale has been used in two studies involving breast cancer patients and patients in medical surgical units. The CCAT (C) has only been validated with hospital inpatients, and it is not clear whether this tool can be adapted for use by other patient populations or practice settings. The extent to which the CCATC reflects patients' views of compassion is also unclear as it was based in part on the Spiritual Needs Survey, which, as Burnell [37] explains, was developed and refined in pastors and chaplains as proxies for patients, and has not been used with patients in any published studies. Furthermore, the 
CCAT(C) currently represents an attempt to define the components of compassionate care rather than a measurement scale, as no data describing the use of the CCAT $\odot$ to measure the characteristics of compassionate care in healthcare providers from the patient's perspective appear to be available.

One study identified in this review associated compassion practice with patients' perceptions of quality of care [39]. McClelland and Vogus [39] used a Compassion Practices Scale to identify the effects of facilitators of and barriers to compassionate acts on the ability of healthcare providers to extend compassionate behaviour to patients and their relatives. However, this study was limited by its key-informant design. The extent to which hospitals rewarded compassionate acts or compassionately supported employees was assessed by surveying executives whose experiences may vary from those of healthcare providers or patients, whose compassionate care may be based on nonconditional or altruistic motivators [55]. Despite these limitations, the Compassion Practices Scale was important to include in a review of measures of compassion and compassionate care as it relates compassionate practice with patients' experience of care. Although patients did not directly rate acts of compassionate care in this study, the outcomes reflect the importance and degree of compassionate care provided by healthcare organisations.

This review also discussed evaluation tools that can be used to identify humanistic qualities in medical students, residents and fellows or to enhance healthcare educator instructional performance and quality [40-42]. In these studies, the elements of compassion and compassionate patient care were but one component of a larger assessment of academic performance and clinical assessment. Recruiting, selecting and educating healthcare providers is a complex process involving learner attributes, knowledge, skills and capacity combined with assessment using validated and reliable scoring instruments to measure competency. As deficiencies have been identified in the provision of compassionate care in health systems, it is important to ensure that healthcare students have the ability to nurture their innate capacity for compassion through the education they receive-especially in their clinical training where compassionate care competencies either fail or flourish [56-58].

The Geriatric Attitudes Measure is a scale developed specifically for measuring the attitudes of geriatrics residents and fellows, including 'a compassion toward other people' dimension. It provides a measure of a resident's ability to extend compassion toward older people, and although validated, requires further testing to determine its transferability to other settings [41, 42]. Similarly, the healthcare educator instructional performance assessment identified in this review was directed at assessing instructional quality in an emergency medicine residency in an urban Level I trauma centre in USA [40]. It included an evaluation of instructional performance in patient compassionate care, but the adaptability of this concept to other residencies needs to be investigated. In addition to examining the validity of these specific measures in other settings, we recommend evaluating the transferability of the construct of compassion across different practice settings and cultures to determine whether a universal measure of compassion is feasible or whether context-specific measures need to be developed.

In addition to limitations pertaining to the measurement of compassion in particular, our review revealed important gaps in psychometric evidence regarding measurement validity and reliability. For the majority of the instruments reviewed using the EMPRO, some types of psychometric data were not available. Overall, EMPRO scores are not reported as there were many missing subscale scores. Even the two measures with the highest scores on most subscales, the Schwartz Center Compassionate Care Scale ${ }^{\mathrm{TM}}$ and the Compassion Competence Scale, had little to no evidence regarding responsiveness or interpretability. Two other measures, the CCATC and Compassion Scale, appear to have equally good conceptual foundations, and the Compassion Competence Scale and Schwartz Center Compassionate Care Scale ${ }^{\mathrm{TM}}$ had adequate evidence for some aspects of the measurement model, but there was insufficient evidence to assess one or more of reliability, validity, responsiveness and interpretability. These gaps are owing in part to the instruments each having been used in only one or two studies. They point to the types of psychometric data that are still needed to permit a more thorough assessment. More information is required regarding test-retest reliability, responsiveness, interpretability, burden and use in subpopulations (or evidence that differential performance is not likely) for all instruments. More validity evidence is needed for the Compassion Practices Scale, Compassion Scale and Geriatric Attitudes Measure. Ultimately, more data in all areas would increase confidence in the psychometric properties of each of the instruments.

\section{Conclusion}

This review synthesised the literature related to instruments for measuring aspects of compassionate care within healthcare. The objective was to identify instruments that may be applicable to clinical practice and reveal gaps in the existing evidence base. Each instrument reviewed has different levels of evidence regarding its psychometric properties, that despite limitations, may be useful for specific purposes and should be considered in the development of a psychometrically robust and clinically relevant empirical measure of compassionate care. While 
these instruments measure aspects of compassion, to varying degrees, we could find no single measure that measured the construct in a comprehensive or sufficiently methodologically rigorous fashion. Compassion is challenging to measure, but given the emphasis on patientcentred and compassionate healthcare, a comprehensive instrument for evaluation of compassion in healthcare systems and educational institutions is no longer an option but a necessity. This is particularly important in light of recent reports identifying compassion as a key component of quality care, which healthcare providers, educators and health systems are increasingly obliged to monitor, evaluate and improve upon $[5,11,17,25]$. Due to the diverse nature of healthcare, the various skills that healthcare providers in different disciplines use, and the different patients and families they encounter, it is unlikely that a 'one-size fits all' approach to measuring compassion in healthcare is feasible or desired. Nonetheless, while the dimensions of compassion are expressed uniquely by healthcare providers and experienced individually by patients, this does not preclude research to define, delineate and measure the common components of this complex but integral construct. It is clear that efforts are being made to measure compassion in healthcare; however, there remains an unmet need for a psychometrically validated instrument that is marked by rigour and flexibility, to assess the essential attributes of compassion in diverse populations and healthcare settings.

Author contributions All authors were involved in the study design, overseeing the review process and contributed to the manuscript. In addition to these substantive contributions, SS conceived, designed, oversaw the review and was the primary author of the manuscript. JK and SS conducted the searches and selected eligible studies. LBR and RS conducted the comparative review of the measurement instruments using the EMPRO. TFH provided expert opinion on review content.

\section{Compliance with Ethical Standards}

Funding The preparation of this review was supported by Dr. Sinclair's Cancer Care Research Professorship from the Faculty of Nursing, University of Calgary. Dr. Hack is supported by a Research Chair in Psychosocial and Supportive Care Oncology from the Canadian Breast Cancer Foundation. Dr. Sawatzky is supported by a Canada Research Chair in Patient-Reported Outcomes.

Conflict of interest Drs. Sinclair, Russell, Hack, Kondejewski and Sawatzky have no conflicts of interest pertaining to the conduct or results of this study.

\section{References}

1. Cole-King A, Gilbert P. Compassionate care: the theory and the reality. J Holist Healthc. 2011;8(3):29-37.
2. Goetz J, Keltner D, Simon-Thomas E. Compassion: an evolutionary analysis and empirical review. Psychol Bull. 2010;136(3):351-74.

3. Schantz M. Compassion: a concept analysis. Nurs Forum. 2007;42:48-55.

4. van der Cingel M. Compassion: the missing link in quality of care. Nurse Educ Today. 2014;34:1253-7.

5. Sinclair S, Norris JM, McConnell SJ, et al. Compassion: a scoping review of the healthcare literature. J Pain Symptom Manage BMC Palliat Care. 2016;15:6-20.

6. Sinclair S, McClement S, Raffin Bouchal S, et al. Compassion in healthcare: an empirical model. J Pain Symptom Manage. 2016;51(2):193-203.

7. Frampton SB. Compassion as the foundation of patient-centered care: the importance of compassion in action. J Comp Eff Res. 2013;5(3):443-55.

8. Heyland DK, Dodek P, Rocker G, Canadian Researchers End-ofLife Network (CARENET), et al. What matters most in end-oflife care: perceptions of seriously ill patients and their family members. CMAJ. 2006;174(5):627-33.

9. Riggs JS, Woodby LL, Burgio KL, et al. "Don't get weak in your compassion": bereaved next of kin's suggestions for improving end-of-life care in Veterans Affairs Medical Centers. J Am Geriatr Soc. 2014;62(4):642-8.

10. Crowther J, Wilson KC, Horton S, Lloyd-Wiliams M. Compassion in healthcare: lessons from a qualitative study of the end of life care of people with dementia. J R Soc Med. 2013;106(12):492-7.

11. Francis R. Report of the Mid Staffordshire NHS Foundation Trust public inquiry. London: The Stationary Office; 2013.

12. Flocke SA, Miller WL, Crabtree BF. Relationships between physician practice style, patient satisfaction, and attributes of primary care. J Fam Pract. 2002;51(10):835-40.

13. McDonagh JR, Elliott TB, Engelberg RA, et al. Family satisfaction with family conferences about end-of-life care in the intensive care unit: increased proportion of family speech is associated with increased satisfaction. Crit Care Med. 2004;32(7):1484-8.

14. Lown BA, Rosen J, Marttila J. An agenda for improving compassionate care: a survey shows about half of patients say such care is missing. Health Aff (Millwood). 2011;30:1772-8.

15. Youngsen R. Foreward. In: Shea S, Wynyard R, Lioni C, editors. Providing compassionate healthcare: challenges in policy and practice. London: Routledge; 2014. pp. xix-xxiii.

16. Sturgeon D. Measuring compassion in nursing. Nurs Stand. 2008;22(46):42-3.

17. Willis L. Raising the bar: the shape of caring review. London: Health Education England; 2015.

18. Paterson R. Can we mandate compassion? Hastings Cent Rep. 2011;41(2):20-3.

19. Institute of Medicine. Improving medical education: enhancing the behavioral and social science content of medical school curricula. Washington, DC: National Academies; 2004.

20. American Medical Association. Code of medical ethics: principle 1 2001. Available from: http://www.ama-assn.org/ama/pub/ physician-resources/medical-ethics/code-medical-ethics/principlesmedical-ethics.page. Accessed Feb 2016.

21. Maclean R. The Vale of Leven Hospital Inquiry. Edinburgh: APS Group; 2014.

22. Eva K, Rosenfeld J, Reiter H, Norman G. An admissions OSCE: the multiple mini interview. Med Educ. 2004;38:314-26.

23. Callwood A, Cooke D, Allan H. Developing and piloting the multiple mini-interview in pre-registration student midwife selection in a UK setting. Nurse Educ Today. 2014;34:1450-4.

24. Department of Health. Confidence in caring: a framework for best practice. London: Department of Health; 2008. 
25. National Health Service Commissioning Board Chief Nursing Officer and Department of Health Chief Nursing Adviser. Compassion in practice: nursing, midwifery and care staff. Our vision and strategy. Leeds: Department of Health; 2012.

26. Reader TW, Gillespie A. Patient neglect in healthcare institutions: a systematic review and conceptual model. BMC Health Serv Res. 2013;13:156-70.

27. Papadopoulos I, Ali S. Measuring compassion in nurses and other healthcare professionals: an integrative review. Nurse Educ Pract. 2016;16(1):133-9.

28. Bramley L, Matiti M. How does it really feel to be in my shoes? Patients' experiences of compassion within nursing care and their perceptions of developing compassionate nurses. J Clin Nurs. 2014;23(19-20):2790-9.

29. van der Cingel M. Compassion in care: a qualitative study of older people with a chronic disease and nurses. Nurs Ethics. 2011;18(5):672-85.

30. Sinclair S, Beamer K, Hack TF, et al. Sympathy, empathy, and compassion: a grounded theory study of palliative care patients' understandings, experiences, and preferences. Palliat Med. 2016. doi: $10.1177 / 0269216316663499$.

31. Popay J. Guidance on the conduct of narrative synthesis in systematic reviews: a product from the ESRC Methods Programme. Lancaster: Lancaster University; 2006.

32. Valderas JM, Ferrer M, Mendivil J, et al. Development of EMPRO: a tool for the standardized assessment of patient-reported outcome measures. Value Health. 2008;11(4):700-8.

33. Terwee CB, Jansma EP, Riphagen II, de Vet HCW. Development of a methodological PubMed search filter for finding studies on measurement properties of measurement instruments. Qual Life Res. 2009;18:1115-23.

34. Lee Y, Seomun G. Development and validation of an instrument to measure nurses' compassion competence. Appl Nurs Res. 2016;30:76-82.

35. Fogarty LA, Curbow BA, Wingard JR, et al. Can 40 seconds of compassion reduce patient anxiety? J Clin Oncol. 1999;17(1):371-9.

36. Kret DD. The qualities of a compassionate nurse according to the perceptions of medical-surgical patients. Med Surg Nurs. 2011;20(1):29-36.

37. Burnell L, Agan D. Compassionate care can it be defined and measured? The development of the compassionate care assessment tool. Int J Caring Sci. 2013;6:180-7.

38. Lown BA, Muncer SJ, Chadwisk R. Can compassionate healthcare be measured? The Schwartz Center Compassionate Care Scale $^{\mathrm{TM}}$. Patient Educ Couns. 2015;98(8):1005-10.

39. McClelland LE, Vogus TJ. Compassion practices and HCAHPS: does rewarding and supporting workplace compassion influence patient perceptions? Health Serv Res. 2014;49(5):1670-83.

40. Colletti JE, Flottemesch TJ, O'Connell TA, et al. Developing a standardized faculty evaluation in an emergency medicine residency. J Emerg Med. 2010;39(5):662-8.

41. Lee M, Reuben DB, Ferrell BA. Multidimensional attitudes of medical residents and geriatrics fellows toward older people. J Am Geriatr Soc. 2005;53:489-94.
42. Reuben DB, Fullerton JT, Tschann JM, Croughan-Minihane M. Attitudes of beginning medical students toward older persons: a five-campus study. J Am Geriatr Soc. 1995;43:1430-6.

43. Park T, Lee HJ. The effects of public officials' sociological variables on their emotional competence in the Korean central government. Korea Public Admin Rev. 2011;45:1-26.

44. Sprecher S, Fehr B. Compassionate love for close others and humanity. J Soc Pers Relat. 2005;22:629-51.

45. Davis MH. Measuring individual differences in empathy: evidence for a multidimensional approach. J Pers Soc Psychol. 1983;44:113-26.

46. Galek K, Flannelly K, Vane A, Galek R. Assessing a patient's spiritual needs: a comprehensive instrument. Holist Nurs Pract. 2005;19:62-9.

47. Wu Y, Larrabee JH, Putman HP. Caring behaviors inventory: a reduction of the 42-item instrument. Nurs Res. 2006;55:18-25.

48. Dewar B. Valuing compassion through definition and measurement. Nurs Manag. 2011;17(9):32-7.

49. McCarthy B. Patients' perceptions of how healthcare providers communicate with them and their families following a diagnosis of colorectal cancer and undergoing chemotherapy treatment. Eur J Oncol Nurs. 2014;18:452-8.

50. Cameron RA, Mazer BL, DeLuca JM, et al. In search of compassion: a new taxonomy of compassionate physician behaviours. Health Expect. 2015;18:1672-85.

51. Way D, Tracy SJ. Conceptualizing compassion as recognizing, relating and (re)acting: a qualitative study of compassionate communication at hospice. Commun Monogr. 2012;79(3):292-315.

52. Netemeyer RG, Bearden WO, Sharma S. Scaling procedures: issues and applications. Thousand Oaks: Sage; 2003. p. 224.

53. DeVellis RF. Scale development: theory and applications. 3rd ed. Newbury Park: Sage; 2012. p. 205.

54. Streiner DL, Norman GR, Cairney J. Health measurement scales: a practical guide to their development and use. 5th ed. Oxford: Oxford University Press; 2015.

55. Vogus TJ, McClelland LE. When the customer is the patient: lessons from healthcare research on patient satisfaction and service quality ratings. Hum Resour Manage Rev. 2016;26(1):37-49.

56. Murphy $\mathrm{F}$, Jones $\mathrm{S}$, Edwards $\mathrm{M}$, et al. The impact of nurse education on the caring behaviours of nursing students. Nurse Educ Today. 2009;29(2):254-64.

57. Bray L, O'Brien MR, Kirton J, et al. The role of professional education in developing compassionate practitioners: a mixed methods study exploring the perceptions of health professionals and pre-registration students. Nurse Educ Today. 2014;34(3):480-6.

58. Smith S, Gentleman M, Loads D, Pullin S. An exploration of a restorative space: a creative approach to reflection for nurse lecturer's focused on experiences of compassion in the workplace. Nurse Ed Today. 2014;24(9):1225-31. 